\title{
Temperature-Modulated DSC Applied to the Transformation Kinetics of Polymer Crystallization
}

\author{
Akihiko Toda, ${ }^{\dagger}$ Takeshi Arita, Chiyoko Tomita, \\ and Masamichi HikosaKa \\ Faculty of Integrated Arts and Sciences, Hiroshima University, \\ 1-7-1 Kagamiyama, Higashi-Hiroshima 739-8521, Japan \\ (Received January 18, 1999)
}

\begin{abstract}
Temperature-modulated differential scanning calorimetry (T-MDSC) has been applied to the isothermal crystallization of poly(vinylidene fluoride), isotactic polypropylene, syndiotactic polypropylene, poly(ethylene terephthalate), poly(caprolactam) and poly(ethylene succinate). It has been confirmed that the imaginary part of the apparent heat capacity determined by T-MDSC gives the temperature dependence of linear growth rate. The proper choice of the baseline for the phase angle of the complex heat capacity has been discussed and it is concluded that the change in the crystallinity evaluated from the integration of mean exothermic heat flow can be used as the baseline.
\end{abstract} KEY WORDS Temperature-Modulated Differential Scanning Calorimetry / Crystallization Kinetics / Phase Angle /

Temperature-modulated differential scanning calorimetry $(\mathrm{T}-\mathrm{MDSC})^{1-3}$ applies a thermal modulation in temperature to a conventional DSC run and determines a dynamic heat capacity from the relationship between the modulation components of temperature and of heat flow. Primary application of this technique is the measurement of specific heat capacity and the examination of the anomaly in a relaxation process such as $\alpha$ process related to the glass transition. ${ }^{4-8}$ The subject of the present paper is the application to the firstorder phase transition of crystallization. The driving force of the transition is supercooling, and hence the modulation in temperature causes the modulation in the kinetics. We have recently proposed an analysis of the transformation kinetics of crystallization and applied to the crystallization of polyethylene and poly(ethylene terephthalate). ${ }^{9-12}$ It has been experimentally confirmed that the temperature dependence of growth rate is obtainable by the analysis. It has been noticed that the important point is the determination of the phase angle in the apparent heat capacity. The determination is influenced by the choice of baseline which is dependent on the heat capacity and thermal conductivity of the sample. Many arguments have been raised on the choice in the glass transition and melting region of polymers. ${ }^{13-15}$ In the present paper, we have examined the crystallization kinetics of several polymers to establish the phase angle correction for polymer crystallization.

In the following, we firstly review our analysis method of crystallization kinetics of polymers with T-MDSC. Secondly, the calibration method of T-MDSC is outlined. The experimental results will be discussed subsequently.

\section{$\operatorname{MODEL}^{9-12}$}

T-MDSC examines the relationship between the modulation components of temperature, $T_{\mathrm{s}}=\bar{T}_{\mathrm{s}}+$ $\operatorname{Re}\left[\tilde{T}_{\mathrm{s}} \mathrm{e}^{i(\omega t+\varepsilon)}\right]$, and of heat flow, $\dot{Q}=\overline{\dot{Q}}+\operatorname{Re}\left[\tilde{\dot{Q}}^{i(\omega t+\delta)}\right]$, and determines the dynamic heat capacity, $\widetilde{\Delta C} \mathrm{e}^{-i \alpha}$, from the relationship, as,

$$
\tilde{\dot{Q}} \mathrm{e}^{i(\omega t+\delta)}=-\widetilde{\Delta C} \mathrm{e}^{-i \alpha} \frac{\mathrm{d}}{\mathrm{d} t} \tilde{T}_{\mathrm{s}} \mathrm{e}^{i(\omega t+\varepsilon)}
$$

The dynamic heat capacity in a relaxation process has a negative imaginary part, and hence $\widetilde{\Delta C} \mathrm{e}^{-i \alpha}$ is usually expressed as,

$$
\widetilde{\Delta C} \mathrm{e}^{-i \alpha} \equiv \widetilde{\Delta C^{\prime}}-i \widetilde{\Delta C^{\prime \prime}}
$$

Owing to the exothermic heat flow of crystallization, $F(t)$, as well as the heat flow due to the heat capacity, the heat flow, $\dot{Q}$, in total is represented as follows,

$$
\dot{Q}=-C_{\mathrm{s}} \frac{\mathrm{d} T_{\mathrm{s}}}{\mathrm{d} t}+F(t)
$$

where $C_{\mathrm{s}}$ is the true heat capacity of the sample.

The driving force of crystallization is the supercooling, $\Delta T$, which is usually larger than $10 \mathrm{~K}$ in the crystallization of most polymers. Therefore, a small modulation in temperature (e.g., $\sim 0.2 \mathrm{~K}$ ) introduces the modulation in supercooling and consequently in the growth rate, as follows,

$$
G\left(T_{\mathrm{s}}\right)=G\left(\bar{T}_{\mathrm{s}}\right)+\frac{\mathrm{d} G\left(\bar{T}_{\mathrm{s}}\right)}{\mathrm{d} T} \tilde{T}_{\mathrm{s}} \mathrm{e}^{i(\omega t+\varepsilon)}
$$

The kinetics of crystallization can be very slow with a proper choice of crystallization temperature (supercooling) and hence the condition of (quasi-)steady state is easily attainable. Under those conditions, the exothermic heat flow of crystallization will have an expansion about temperature modulation, as follows,

$$
F\left(t, T_{\mathrm{s}}\right)=\bar{F}\left(T, \bar{T}_{\mathrm{s}}\right)+F_{T}^{\prime}\left(t, \bar{T}_{\mathrm{s}}\right) \tilde{T}_{\mathrm{s}} \mathrm{e}^{i(\omega t+\varepsilon)}
$$

where $F_{T}^{\prime}$ represents the coefficient of the response to temperature modulation, which will be proportional to the temperature dependence of growth rate in eq 4 . The following relation must be satisfied among those modulation components, 


$$
\tilde{Q} \mathrm{e}^{i(\omega t+\delta)}=-C_{\mathrm{s}} \frac{\mathrm{d}}{\mathrm{d} t} \tilde{T}_{\mathrm{s}} \mathrm{e}^{i(\omega t+\varepsilon)}+F_{T}^{\prime} \widetilde{T}_{\mathrm{s}} \mathrm{e}^{i(\omega t+\varepsilon)}
$$

and hence, when arranged as the heat capacity of eq 1 , the apparent heat capacity is expressed as,

$$
\widetilde{\Delta C} \mathrm{e}^{-i \alpha}=C_{\mathrm{s}}+i \frac{1}{\omega} F_{T}^{\prime}
$$

The following discussion is based on this expression of the apparent heat capacity in transformation.

We discuss the details of the crystallization kinetics. The growth of polymer crystals is understood as the growth of spherulites composed of thin lamellar crystals. When the crystals grow linearly at the rate of $G$ with the total area of the growth face, $S_{\text {total }}$, the exothermic heat flow of crystallization will be represented as follows,

$$
F=\Delta h_{\mathrm{f}} S_{\text {total }} G
$$

where $\Delta h_{\mathrm{f}}$ represents the heat of fusion per unit volume of crystals. The temperature coefficient of the exothermic heat flow is then given as,

$$
F_{T}^{\prime}(t)=\Delta h_{\mathrm{f}} S_{\text {total }}(t) \frac{\mathrm{d} G}{\mathrm{~d} T}=\bar{F}(t) \frac{\mathrm{d}}{\mathrm{d} T} \ln \left(G / G_{0}\right)
$$

The apparent heat capacity on crystallization is therefore given as,

$$
\widetilde{\Delta C} \mathrm{e}^{-i \alpha}=C_{\mathrm{s}}+i \frac{1}{\omega} \vec{F}(t) \frac{\mathrm{d}}{\mathrm{d} T} \ln \left(G / G_{0}\right)
$$

On the other hand, it has been experimentally confirmed that the frequency dependence of the apparent heat capacity is expressed as,

$$
\widetilde{\Delta C} \mathrm{e}^{-i \alpha}=A+i \frac{B}{\omega}
$$

where $A$ and $B$ are constants. ${ }^{10,12}$ Therefore, the real part of the apparent heat capacity corresponds to $C_{\mathrm{s}}$ and the imaginary part to $\bar{F}(\mathrm{~d} / \mathrm{d} T) \ln \left(G / G_{0}\right)$ in eq 10 . From the imaginary part, $\widetilde{\Delta C}=\widetilde{\Delta C} \sin \alpha$, and the mean exothermic heat flow, $\bar{F}$, eq 10 gives the temperature dependence of growth rate as follows,

$$
\frac{d}{\mathrm{~d} T} \ln \left(G / G_{0}\right)=-\frac{\omega \widetilde{\Delta C} \sin \alpha}{\bar{F}}
$$

As shown in the above expression, the phase angle, $\alpha$, of the complex heat capacity needs to be determined precisely for the analysis.

\section{CALIBRATION METHOD}

It has been pointed out that the magnitude and phase angle of the complex heat capacity obtained by T-MDSC need to be calibrated owing to the thermal property (i.e., heat capacity and thermal conductance) of the instrument. ${ }^{13,16-20}$ It is known that the (apparent) heat capacity of sample influences the calibration constant and complicates the calibration. The situation is typically represented by Hatta's model ${ }^{19}$ shown in Figure 1. The model is expressed by the following equations,

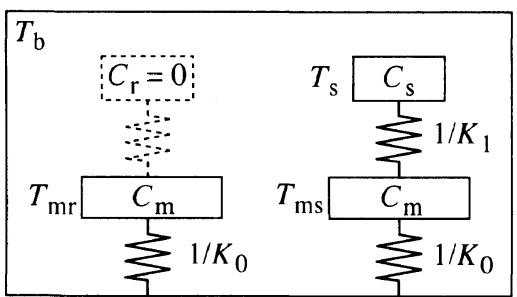

Figure 1. Schematic representation of Hatta's model ${ }^{19}$ of T-MDSC without reference pan. In the figure, $T_{\mathrm{b}}, T_{\mathrm{rm}}$, and $T_{\mathrm{sm}}$ represent the temperatures of the block, reference and sample monitoring stations, $C_{\mathrm{m}}$ and $C_{\mathrm{s}}$ the heat capacity of the monitoring station and sample (+ sample pan) and $K_{0}$ and $K_{1}$ the Newton's law constants of heat transfer between the block and monitoring station and between the monitoring station and sample or reference pan, respectively.

$$
\begin{gathered}
C_{\mathrm{m}} \dot{T}_{\mathrm{rm}}=K_{0}\left(T_{\mathrm{b}}-T_{\mathrm{rm}}\right) \\
C_{\mathrm{m}} \dot{T}_{\mathrm{sm}}=K_{0}\left(T_{\mathrm{b}}-T_{\mathrm{sm}}\right)+K_{1}\left(T_{\mathrm{s}}-T_{\mathrm{sm}}\right) \\
C_{\mathrm{s}} \dot{T}_{\mathrm{s}}-F=K_{1}\left(T_{\mathrm{sm}}-T_{\mathrm{s}}\right)
\end{gathered}
$$

where $T_{\mathrm{b}}, T_{\mathrm{rm}}$, and $T_{\mathrm{sm}}$ represent the temperatures of the block, reference and sample monitoring stations, $C_{\mathrm{m}}$ the heat capacity of the monitoring station and $K_{0}$ and $K_{1}$ the Newton's law constants of thermal conductance between the block and monitoring station and between the monitoring station and sample or reference pan, respectively. With T-MDSC of heat flux type, we obtain the temperature at the sample monitoring station, $T_{\mathrm{sm}}=\bar{T}_{\mathrm{sm}}+\operatorname{Re}\left[\bar{T}_{\mathrm{sm}} \mathrm{e}^{i(\omega t+\varepsilon)}\right]$ and the heat flow, $\dot{Q}$, calculated from the temperature difference, $T_{\mathrm{sm}}-T_{\mathrm{rm}}$ : $\dot{Q}=\overline{\dot{Q}}+\operatorname{Re}\left[\tilde{\dot{Q}}^{i(\omega t+\delta)}\right] \equiv K_{0}\left(T_{\mathrm{sm}}-T_{\mathrm{rm}}\right)$.

The raw data of the heat capacity is defined as,

$$
C_{0} \mathrm{e}^{-i \varphi} \equiv \frac{\tilde{Q}}{\omega \tilde{T}_{\mathrm{sm}}} \mathrm{e}^{-i(\varepsilon-\delta-\pi / 2)}
$$

and the (apparent) heat capacity, $\widetilde{\Delta C} \mathrm{e}^{-i \alpha}$, of eq 7 is related with the raw data as follows,

$$
\begin{gathered}
\frac{\widetilde{\Delta C} \mathrm{e}^{-i \alpha}}{C_{0} \mathrm{e}^{-i \varphi}}=\left(1+i \omega \tau_{0}\right)\left(1+i \omega \tau_{1}\right) \\
\tau_{0} \equiv \frac{C_{\mathrm{m}}}{K_{0}} \\
\tau_{1} \equiv \frac{\widetilde{\Delta C \mathrm{e}^{i \alpha}}}{K_{1}}
\end{gathered}
$$

Therefore, the calibration constant is dependent on the apparent heat capacity, $\widetilde{\Delta C} \mathrm{e}^{-i \alpha}$ in $\tau_{1}$. When $F_{T}^{\prime}=0$ (no transformation: $\widetilde{\Delta C} \mathrm{e}^{i \alpha}=C_{\mathrm{s}}$ and $\alpha=0$ ) and $\omega \tau_{1}, \omega \tau_{2}$ $\ll 1$, the magnitude and the phase angle are expressed as,

$$
\begin{aligned}
\left|\frac{C_{\mathrm{s}}}{C_{0}}\right|^{2} & \cong 1+\omega^{2}\left(\tau_{0}^{2}+\frac{C_{\mathrm{s}}^{2}}{K_{1}^{2}}\right) \\
\varphi & \cong \omega\left(\tau_{0}+\frac{C_{\mathrm{s}}}{K_{1}}\right)
\end{aligned}
$$

and it is demonstrated that the raw data of the phase angle, $\varphi$, is an increasing function of $C_{\mathrm{s}} / K_{1}$.

When polymer crystallization is concerned, it has been found that the heat capacity changes in an opposite way depending on the crystallization temperature. ${ }^{9-12}$ At relatively lower temperature near the glass transition, the 
heat capacity monotonously decreases with the progress of crystallization, while at higher temperature near the melting point the heat capacity increases on crystallization and then shows a gradual decrease when kept at the same temperature. Though the physical meaning of the initial increase has not been well clarified, those changes in the heat capacity will introduce the opposite effect on the baseline of the phase angle; the increase (decrease) in heat capacity will result in the increase (decrease) in the phase angle as shown in eq 21 . In the following experiments, we will examine the change in the baseline of the phase angle with several polymers and discuss the proper method of baseline subtraction for the determination of the temperature dependence of growth rate.

\section{EXPERIMENTAL}

The DSC 2920 Module controlled with Thermal Analyst 2200 (TA Instruments) was used for all measurements. Helium gas with a flow rate of $40 \mathrm{~mL} \mathrm{~min}^{-1}$ was purged through the cell. The samples were poly(vinylidene fluoride) (PVDF, Kureha Chemicals Inc., KF1000), isotactic polypropylene (iPP, Scientific Polymer Products, Inc.), syndiotactic polypropylene (sPP, Mitsui Chemicals Inc.), poly(ethylene terephthalate) (PET, Toyobo Co., Ltd.), poly(caprolactam) (Nylon 6, Scientific Polymer Products, Inc.) and poly(ethylene succinate) (PeSu, Scientific Polymer Products, Inc.). We prepared disk-shaped thin films of the samples the weight of which was in the range of $3.2-7.7 \mathrm{mg}$. It was confirmed that there was no qualitative change by the sample weight in this range. Crystallization was examined under quasi-isothermal condition around a fixed temperature. When crystallized near the glass transition of PET, Nylon 6, and PeSu, the sample was firstly quenched from the melt below the glass transition temperature before placing in the DSC apparatus and then heated up to the crystallization temperature in the apparatus. At higher temperatures near the melting point, the samples were directly cooled down from the melt to the crystallization temperature at the rate faster than $20 \mathrm{Kmin}^{-1}$ in the apparatus. The modulation period of $40 \mathrm{~s}$ was examined with the modulation amplitude of $\pm 0.2 \mathrm{~K}$.

Because we are interested in the change in the phase angle, it is preferable to increase the phase angle of the raw data. To do so, an aluminum thin sheet was placed in the reference pan to reduce the difference in the heat capacity between sample and reference. It was confirmed that the results of the analysis with the aluminum thin sheet in the reference pan and without it were essentially identical.

For the optical observation of the growth of spherulites, we employed a polarizing microscope (Olympus BH2) with a hot stage (Linkam THMS600). The hot stage was equipped with a cooling device (L-600A) and the sample could be cooled down to the crystallization temperature from the melt at $130 \mathrm{~K} \mathrm{~min}^{-1}$. From the linear growth rate shown in Figure 2, the temperature dependence of growth rate was determined for PVDF, iPP, PET, and PeSu. In the case of sPP and Nylon 6, nucleation density of the samples was too high to observe the growth of spherulites, and hence the growth

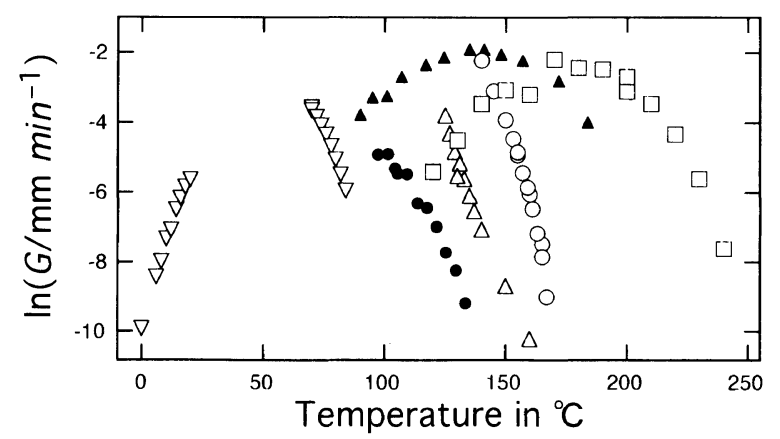

Figure 2. Linear growth rate of spherulites plotted against crystallization temperature: $O$, PVDF; $\triangle$, iPP; $\mathbf{O}$, sPP; $\square$, PET; $\mathbf{\Delta}$, Nylon $6 ; \nabla, \mathrm{PeSu}$. The data of PVDF, iPP, PET, and PeSu are the present experimental results of optical microscopy. For sPP and Nylon 6 , the data are taken from ref 21 and 22 .

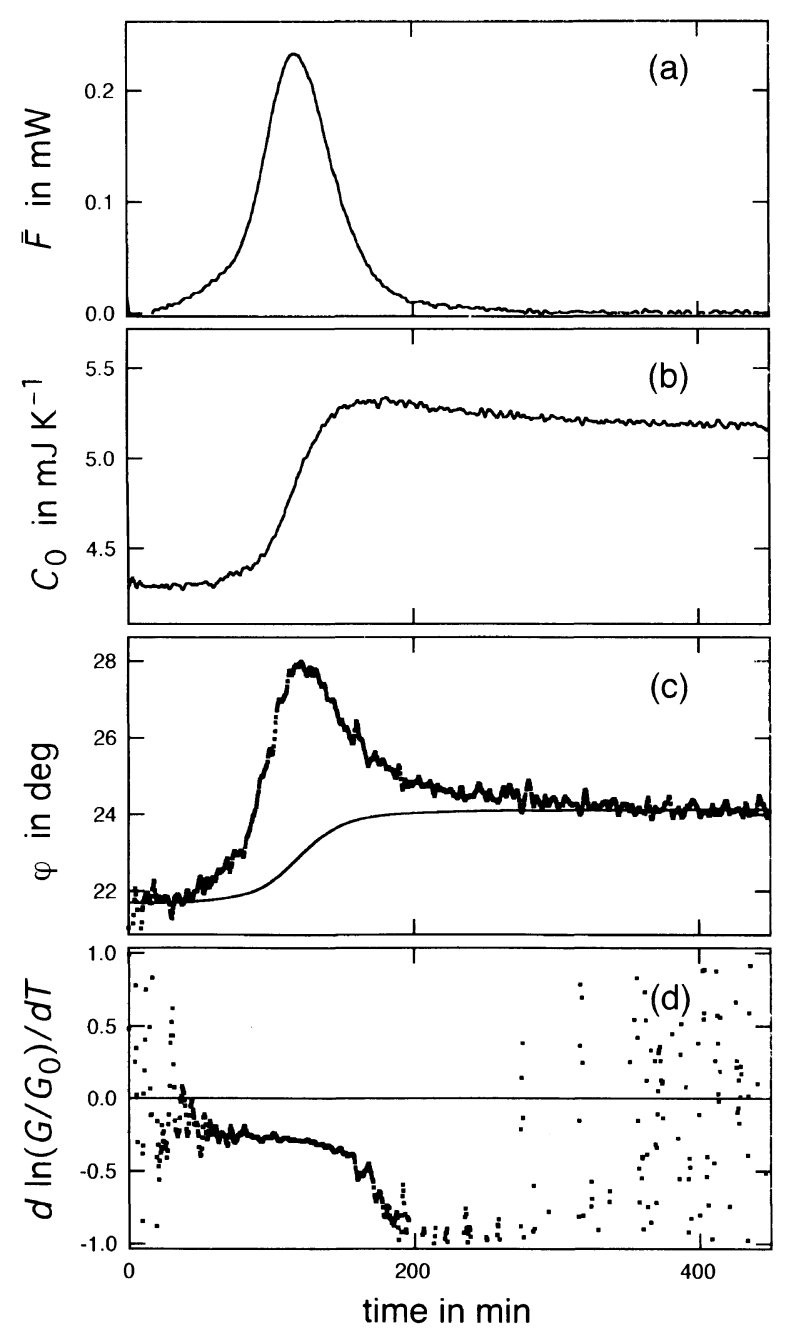

Figure 3. Time sequence of quasi-isothermal crystallization of PVDF from the melt at $158.9^{\circ} \mathrm{C}$ : (a), the mean exothermic heat flow $\bar{F}$; (b), $C_{0} ;(\mathrm{c}), \varphi ;(\mathrm{d}),(\mathrm{d} / \mathrm{d} T) \ln \left(G / G_{0}\right)$ determined by eq 12 . Equation 16 defines $C_{0}$ and $\varphi$ in (b) and (c).

rate data were taken from ref 21 and 22 .

\section{RESULTS AND DISCUSSION}

Figures 3 and 4 show $(\mathrm{a}-\mathrm{c})$ the raw data and (d) the results of the analysis on quasi-isothermal crystallization of PVDF and PET, respectively. For PVDF, Figure 3 


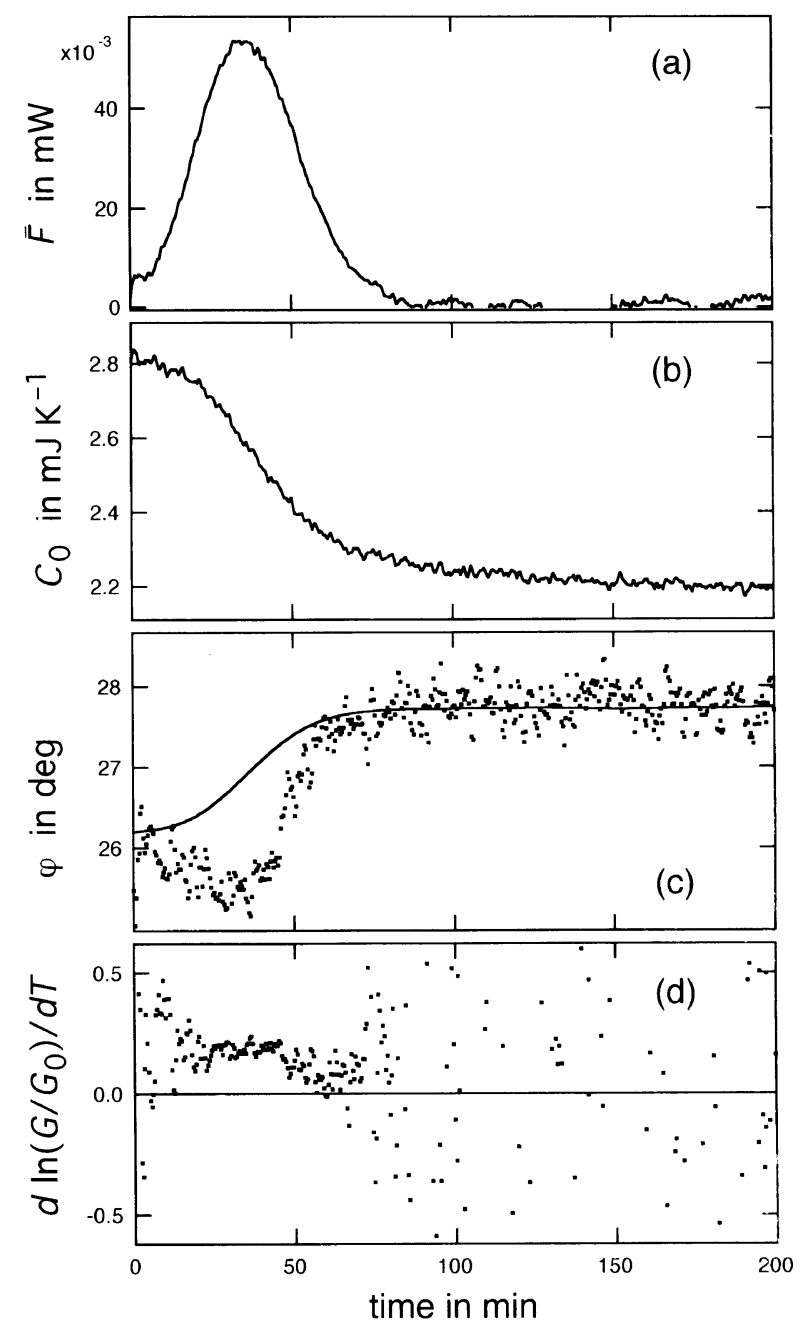

Figure 4. Time sequence of quasi-isothermal crystallization of PET from the melt at $111.1^{\circ} \mathrm{C}$ : (a), the mean exothermic heat flow $\bar{F}$; (b), $C_{0} ;(\mathrm{c}), \varphi ;(\mathrm{d}),(\mathrm{d} / \mathrm{d} T) \ln \left(G / G_{0}\right)$ determined by eq 12 .

shows the results of an ordinary crystallization near the melting point, while Figure 4 shows the results of cold crystallization near the glass transition temperature of PET. It is clearly demonstrated in Figures $3 \mathrm{~b}$ and $4 \mathrm{~b}$ that the heat capacity monotonously decreases with the progress of cold crystallization, while the heat capacity increases on the ordinary crystallization and then shows a gradual decrease when kept at the same temperature. On the other hand, the phase angle exhibits a peak in the ordinary crystallization and a negative peak (dip) in the cold crystallization as seen in Figures $3 \mathrm{c}$ and $4 \mathrm{c}$. This difference in the direction of the change is due to the fact that the temperature dependence of growth rate changes its sign for the ordinary crystallization and cold crystallization, as seen in the bell shaped curves of the growth rate in Figure 2 for PET, PeSu, and Nylon 6.

The baseline of the phase angle shifts upward on crystallization for both crystallization modes, even though the heat capacity shows an opposite change. This was also the case for other polymers examined. As discussed in the section of "Calibration Method", the increase (decrease) in the heat capacity will bring the increase (decrease) in the baseline of phase angle. Therefore, the increase in the baseline for the cold
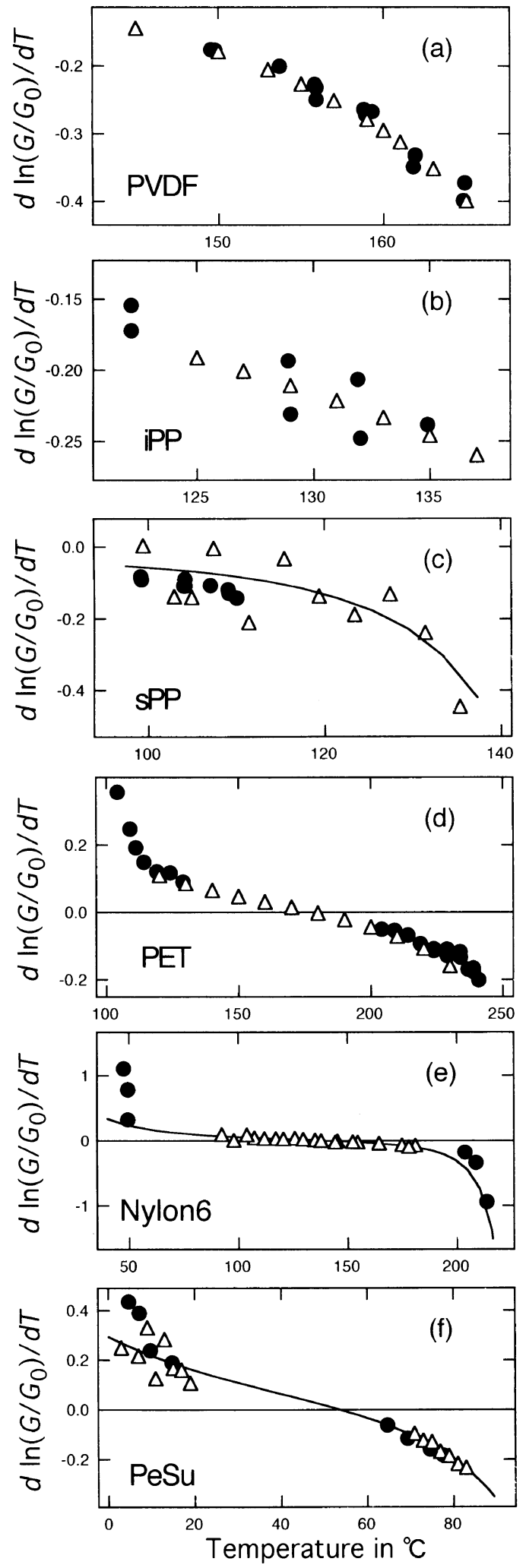

Figure 5. Plots of the temperature dependence, $(\mathrm{d} / \mathrm{d} T) \ln \left(G / G_{0}\right)$. The T-MDSC results $(-)$ were determined by eq 12 . The data $(\triangle)$ are calculated from the results of direct measurements by microscopy shown in Figure 2.

crystallization cannot be due to the change in the magnitude of the heat capacity which decreases monotonously. As an alternative possibility, thermal contact between the sample and the sample cell is expected to become worse on crystallization, and the heat transfer coefficient similar to $K_{1}$ in Hatta's model of eq 21 
may become smaller. Consequently, eq 21 predicts the upward shift of the baseline on crystallization.

In Figures $3 c$ and $4 c$, the baseline was approximated by the integrated exothermic heat flow (i.e., by the change in crystallinity) whose width was fitted to the total change in the baseline before and after the crystallization. With this baseline, the temperature dependence of growth rate keeps a constant value around the peak of the mean exothermic heat flow, as shown in Figures $3 \mathrm{~d}$ and $4 \mathrm{~d}$; we expect steady and linear growth of crystals around the peak.

Figure 5 shows the experimental results of T-MDSC (i.e., the values of $(\mathrm{d} / \mathrm{d} T) \ln \left(G / G_{0}\right)$ near the peak of the exotherm). The results are compared with the results of direct observation by optical microscopy. The agreement between those independent results justifies the choice of the baseline shown in Figures $3 \mathrm{c}$ and $4 \mathrm{c}$.

\section{CONCLUSION}

We have successfully demonstrated the applicability of T-MDSC to polymer crystallization in the determination of the temperature dependence of growth rate. This method is not influenced by the details of the kinetics (e.g., number density of nuclei) and hence it will be a unique method to determine the temperature dependence, when the optical observation of crystallization is not available.

The phase angle correction of the complex heat capacity is necessary because of the upward shift of baseline on crystallization. The shift is not explained by the change in the sample heat capacity which can increase or decrease depending on the crystallization condition. The reason for the insensitiveness to the change in the heat capacity will be the small change in the heat capacity. The shift in phase angle has been successfully calibrated with the change in crystallinity evaluated from the integration of the mean exothermic heat flow. The shift is probably due to the change in the thermal contact between the sample and sample pan on solidification.

It is known that, in the melting region, the apparent heat capacity becomes more than several times as large as the heat capacity outside the region,,$^{23-25}$ and hence the change in phase angle caused by the change in heat capacity is not negligible. On the other hand, the shift in phase angle caused by the thermal contact is quite small ( $\sim 2$ degrees) as shown in Figures 3 and 4 , and hence the change caused by the thermal contact will be neglected in the melting region where the phase angle has a large peak which becomes several tens degrees. $^{23-25}$
Acknowledgments. The authors thank Messrs. K. Sasaki and C. Nonomura (TOYOBO Co., Ltd.) for the kind supply of the amorphous poly(ethylene terephthalate) films and Dr. Fujio (Mitsui Chemicals Inc.) for the supply of syndiotactic polypropylene. This work was partly supported by Grants-in-Aid for Scientific Research from the Ministry of Education, Science, Sports and Culture of Japan and by NEDO International Joint Research Program.

\section{REFERENCES}

1. P. S. Gill, S. R. Sauerbrunn, and M. Reading, J. Therm. Anal., 40, 931 (1993).

2. M. Reading, D. Elliott, and V. I. Hill, J. Therm. Anal., 40, 949 (1993).

3. M. Reading, A. Luget, and R. Wilson, Thermochim. Acta, 238 295 (1994).

4. N. O. Birge and S. R. Nagel, Phys. Rev. Lett., 54, 2674 (1985).

5. Y. H. Jeong and I. K. Moon, Phys. Rev. B, 52, 6381 (1995).

6. A. Hensel, J. Dobbertin, J. E. K. Schawe, A. Boller, and C. Schick, J. Therm. Anal., 46, 935 (1996).

7. B. Wunderlich and I. Okazaki, in "Structure and Properties of Glassy Polymers", M. R. Tant and A. J. Hill, Ed., ACS Symposium Series, Am. Chem. Soc., Washington, D.C., to be published.

8. A. Toda, C. Tomita, and M. Hikosaka, Prog. Theor. Phys. Suppl., 126, 103 (1997)

9. A. Toda, T. Oda, M. Hikosaka, and Y. Saruyama, Polymer, 38, 231 (1997).

10. A. Toda, T. Oda, M. Hikosaka, and Y. Saruyama, Thermochim Acta, 293, 47 (1997).

11. A. Toda, C. Tomita, M. Hikosaka, and Y. Saruyama, Polymer, 38, 2849 (1997).

12. A. Toda, C. Tomita, M. Hikosaka, and Y. Saruyama, Polymer, 39, 1439 (1998).

13. S. Weyer, A. Hensel, and C. Schick, Thermochim. Acta, 305, 267 (1997).

14. G. Van Assche, A. Van Hemelrijck, and B. Van Mele, J. Therm. Anal., 49, 443 (1997).

15. Z. Jiang, C. T. Imrie, and J. M. Hutchinson, Thermochim. Acta, 315, 1 (1998).

16. B. Wunderlich, Y. Jin, and A. Boller, Thermochim. Acta, 238, 277 (1994).

17. A. Boller, Y. Jin, and B. Wunderlich, J. Therm. Anal., 42, 307 (1994).

18. I. Hatta, Jpn. J. Appl. Phys., 33, L686 (1994).

19. I. Hatta and S. Muramatsu, Jpn. J. Appl. Phys., 35, L858 (1996)

20. J. E. K. Schawe and G. W. H. Höhne, J. Therm. Anal., 46, 893 (1996).

21. R. L. Miller and E. G. Seeley, J. Polym. Sci., Polym. Phys. Ed., 20, 2297 (1982).

22. J.H. Magill, Polymer, 6, 367 (1965).

23. A. Toda, C. Tomita, M. Hikosaka, and Y. Saruyama, Polymer, 39, 5093 (1998).

24. A. Toda, C. Tomita, M. Hikosaka, and Y. Saruyama, Thermochim. Acta, 324, 95 (1998).

25. A. Toda, C. Tomita, and M. Hikosaka, J. Therm. Anal., 51, 623 (1998). 